\title{
Low-frequency acoustic emissions in fresh and salt water
}

\author{
Ali R. Kolaini \\ National Center for Physical Acoustics, University of Mississippi, University, Mississippi 38677 \\ Ronald A. Roy \\ Applied Physics Laboratory, University of Washington, Seattle, Washington 98105 \\ David L. Gardner \\ NOAA, PMEL, 7600 Sound Point Way, Seattle, Washington 98115
}

(Received 18 December 1993; accepted for publication 5 May 1994)

The impact of a jet of water onto a still water surface results in the entrainment of large amounts of air and the eventual formation of a bubble plume. Densely populated bubble plumes are generated by dropping a fixed volume of water, held in a cylindrical container, onto a still-water surface. The detached bubble plume, which is roughly spherical in shape, then undergoes volume pulsation and radiates relatively large-amplitude, low-frequency sound. The results of a laboratory study of the noise produced by this process were reported previously by Kolaini et al. [J. Acoust. Soc. Am. 94, 2809-2820 (1993)]. In this paper we report the results of a field study of noise produced by this process in both fresh water (Lake Washington, WA) and salt water (Puget Sound, WA). Studies of acoustic emissions from transient bubble plumes as a function of cylinder parameters are described, with specific attention devoted to a comparison of the results obtained in salt and fresh water. The measurements, which exhibit good agreement with laboratory study, indicate that there is a correlation between the acoustic intensity radiated from bubble plumes and the total potential energy of the water jet.

PACS numbers: $43.30 . \mathrm{Nb}$

\section{INTRODUCTION}

Some of the earliest measurements of ambient-sound spectral levels in the ocean were reported by Knudsen et al. (1948). These measurements showed that the sound spectral level depends on wind speed and decreases with increasing frequency at the rate of approximately $5 \mathrm{~dB} /$ oct. Later, Wenz (1962) reviewed the data from Knudsen et al. (1948) and concluded that gas bubbles generated by breaking waves are a major contributor to underwater noise. Two recent conferences have been held during which many mechanisms for underwater ambient noise were discussed in some detail (Kerman, 1988, 1993).

Kerman (1984) and Wilson (1983) observed that broadband ambient noise source levels are wind speed and whitecap dependent. Wilson (1983) concluded that there are three distinct noise regimes: (1) prior to wave breaking; (2) after wave breaking with some whitecap index; (3) fully developed sea states with wind speeds greater than $6 \mathrm{~m} / \mathrm{s}$. The results of the measurements of low-frequency noise generated by breaking waves at sea by Chapman and Cornish (1993) indicate that there exist two wind-speed-dependent regimes. Their results suggest that the noise process in breaking waves may be different at low frequencies from that of high frequencies.

The sources of sound production at frequencies $<500$ $\mathrm{Hz}$ are not yet known. Prosperetti (1988) and Carey and Wagstaff (1988) hypothesized, independently, that the collective oscillations of bubble clouds or plumes may be the source of low-frequency ambient noise. [The bubble clouds that can oscillate collectively was previously established by d'Agostino and Brennen (1988).] Carey et al. (1993) exam- ined the role of collective oscillations of bubble plumes produced by simulating breaking waves generated using a water-filled tipping trough.

This experiment was designed to simulate a breaking wave by dropping a cylindrical volume of water onto a free water surface. The bubble plumes so generated, after detachment, oscillate and produce sound based on the average mechanical properties of the mixture. A detailed laboratory study of the acoustic characteristic of bubble plumes generated by impacting transient water jets was reported earlier (Kolaini et al., 1991, 1993). In the present study, the acoustic characteristics of similar bubble plumes generated in the free field in both fresh water (Lake Washington) and salt water (Puget Sound) are examined.

\section{EXPERIMENTAL ARRANGEMENT}

The measurements reported here were carried out in Lake Washington $(0.7 \% \mathrm{NaCl})$ and in Puget Sound $(4.9 \%$ $\mathrm{NaCl})$. The two sites provided nearly free-field conditions with minimal acoustic boundary interaction. The test areas were approximately $15 \mathrm{~m}$ deep with a gently sloping mud bottom and $200 \mathrm{~m}$ from the shore. The bottom-reflected signal can accounted for using (Urick, 1983)

$$
\mathrm{TL}=20 \log (2 r)+20 \log \left(r_{p}\right)
$$

where TL is the transmission loss; the first term and second term represent spherical spreading loss and bottom absorption, respectively. With a hydrophone depth of approximately $1 \mathrm{~m}$ and an assumed bottom reflection loss of $10 \mathrm{~dB}$, the bottom-reflected signal was reduced by a factor of $\sim 40 \mathrm{~dB}$. Since typical signal-to-noise ratios were approximately 35 


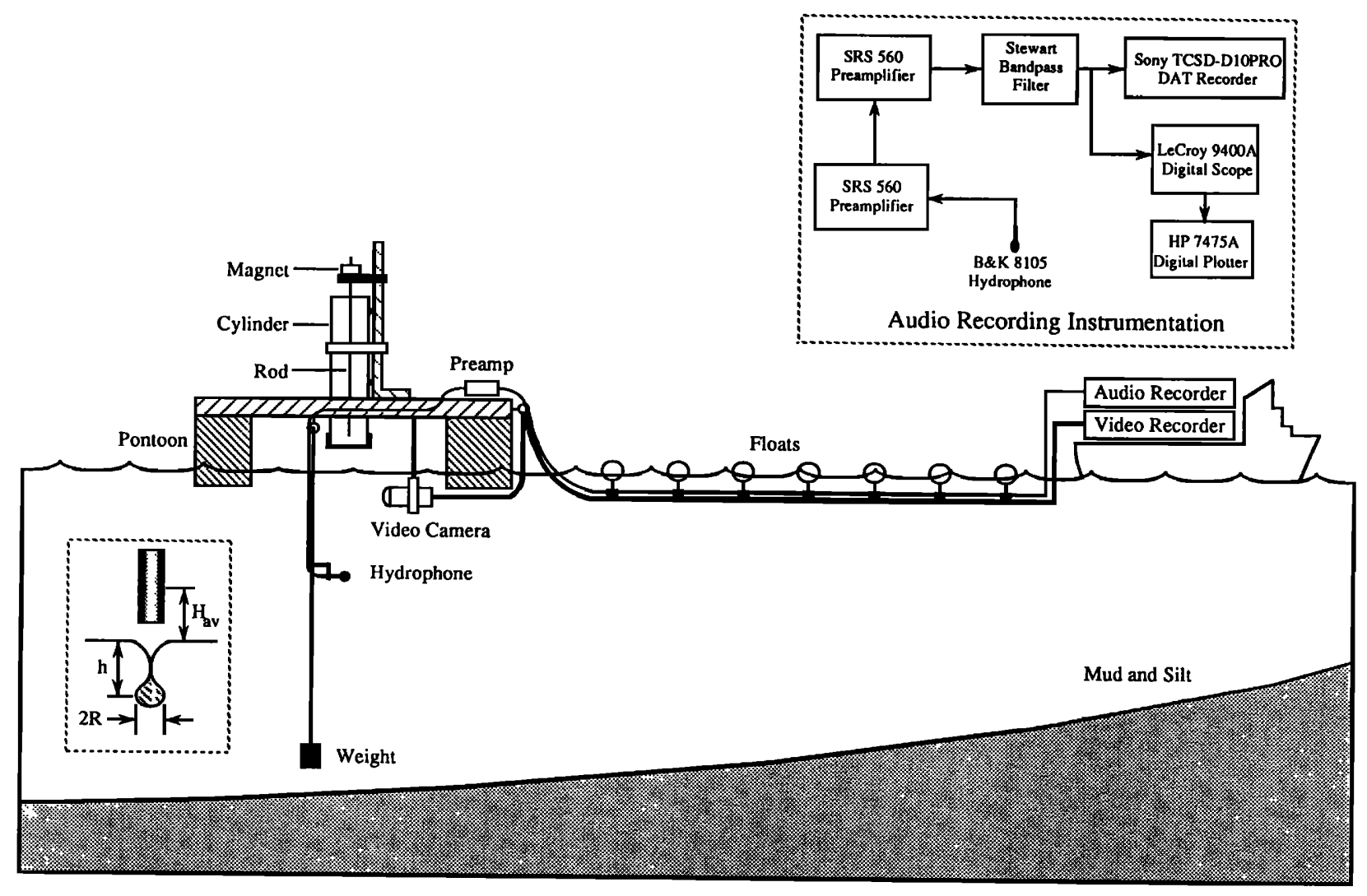

FIG. 1. Schematic drawing of the transient jet experiment specifying the cylinder location, the raft, the vessel, and the instrumentation. The details of the bubble plume generation geometry and the instrumentation are shown in the insertions.

$\mathrm{dB}$, bottom-reflected signals were lost in the noise and did not significantly contribute to the absolute pressure measurements. Occasional small craft passed nearby the test site, but did not interfere with the experiments.

A cylindrical container with an inside diameter of 10.8 $\mathrm{cm}$ was used to release a "slug" of water from heights ranging from 5 to $30 \mathrm{~cm}$ above the still-water surface. The container was filled with water volumes ranging from 0.15 to 4.2 liters. The cylinder was suspended above the sea surface by a floating raft and was restrained from lateral movement. The water column was supported by a tightly stretched, thin rubber sheet which was secured to the bottom of the container by a rubber band. The rubber membrane was ruptured by a thin, sharpened rod, which was coaxially suspended inside the container a few inches above the membrane (see Fig. 1). Once the membrane was ruptured, the cylindrical volume of water fell intact onto the still water surface [for a more detailed description, see Kolaini et al. (1993)].

Air was initially entrained shortly after impact and continued to be entrained along the jet circumference as the entry progressed, forming a cylindrical bubble plume. As the plume grew in length a "neck" formed and the water surrounding this neck moved radially inward. This process continued until the neck collapsed and a bubble plume separated from the crater region caused by the impacting water jet. The detached plume appeared to be a large air-filled "bubble" of radius $R$ with a roughened surface that acted as a mixing layer between the air-filled cavity and the host liquid.

The detached bubble plume underwent volume pulsa- tions which resulted in the production of low-frequency sound. In the laboratory, using high-speed cinematography, we were able to show that large-amplitude, low-frequency sound emission correlated well with bubble plume detachment (Kolaini et al., 1993). Emissions from individual plumes were measured using a B\&K 8105 broadband hydrophone and a Sony TCD-D10PRO digital audio tape recorder. Two Stanford Research Systems SR560 amplifiers/filters, one on the float and one positioned in the launch, provided signal amplification and anti-alias filtering. The digitization speed and conversion gain was $42.1 \mathrm{ksample} / \mathrm{s}$ and $16 \mathrm{bits}$, respectively. A visual record of the evolution of each bubble plume was recorded by an underwater video camera and lighting system affixed to the float.

Experiments consisted of releasing a preset volume of water from a preset height and simultaneously recording the video images and acoustic emissions. Each measurement was duplicated five times, after which either the water volume, water height, or hydrophone location was changed and the entire process repeated. Special care was taken to insure that the rubber membrane was stretched tightly and uniformly. Otherwise portions of the membrane did not completely retract from the cylinder opening and the water poured, rather than dumped, out of the cylinder.

\section{RESULTS AND DISCUSSION}

A series of video images detailing the bubble plume formation process is shown in Fig. 2. These images, which are 


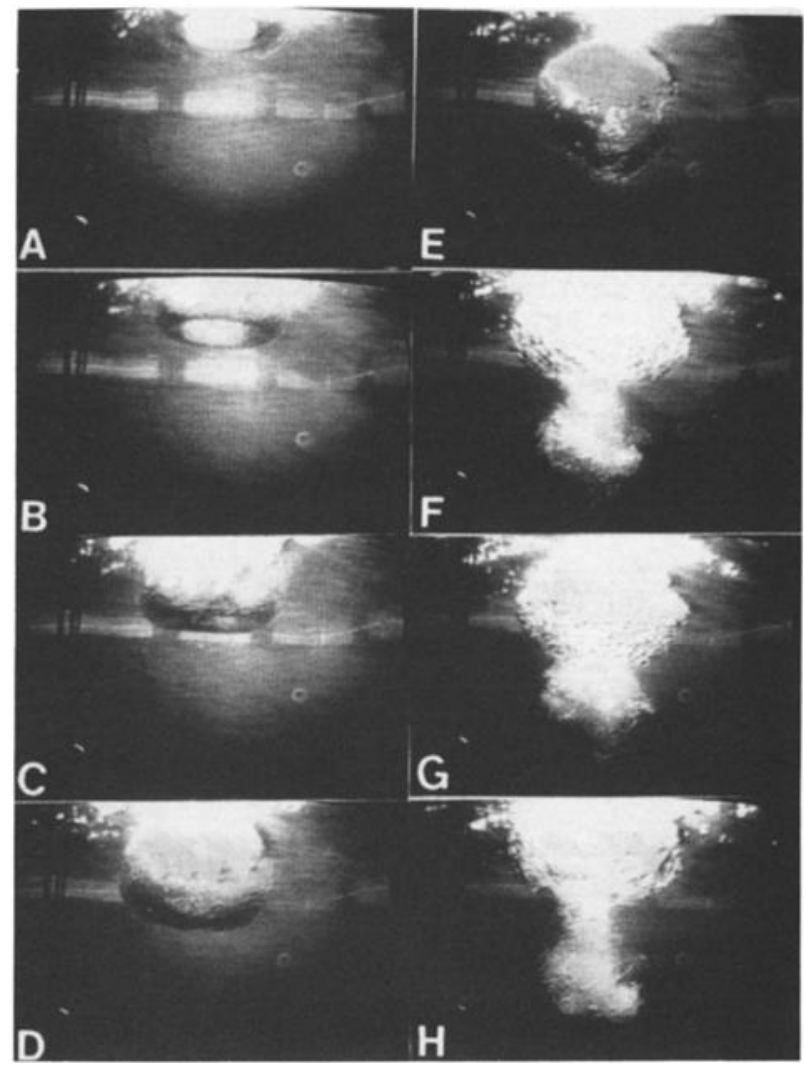

FIG. 2. Photographs of frames taken from an underwater video camera depicting the cylindrical plume growth and the spherical plume detachment in salt water. This plume was generated by releasing a cylindrical volume of water with a radius $5.4 \mathrm{~cm}$, a length of $40 \mathrm{~cm}$, and suspended $15 \mathrm{~cm}$ above the mean water level. The total time interval from frame $\mathrm{A}-\mathrm{H}$ is about 900 ms. The plume size is $10.2 \mathrm{~cm}$ with resonance frequency of $45 \mathrm{~Hz}$.

typical, were obtained by releasing 3.66 liters of Puget Sound water from the cylinder suspended $15 \mathrm{~cm}$ above the calm surface of the Sound. They show the formation of a cylindrical bubble plume (frame $\mathrm{A}-\mathrm{C}$ ) and the subsequent spherical plume detachment (frame $E$ ). The radius of the detached bubble plume was about $10.2 \mathrm{~cm}$ and the radiated noise exhibited a dominant spectral peak at $45 \mathrm{~Hz}$.

It has been shown that, when a bubble plume detaches, there is an axial jet directed downward into the bubble plume and an opposing jet directed upward into the crater formed by the impact. It seems this jet is the physical mechanism that drives the plume into oscillation (Kolaini et al., 1993). [This physical process resembles the excitation of a single bubble released from an underwater nozzle as described by Longuet-Higgins et al. (1991) and a bubble detachment from the crater made by a falling rain-drop reported by Prosperetti et al. (1989) and Oguz and Prosperetti (1991).] The generation of low-frequency sound from a detached plume can be described as that of a bubble cloud that oscillates collectively with a fundamental resonance frequency that scales with the volume of the cloud and the sound speed within the bubbly mixture. The latter is typically related to the free-gas void fraction through a form of Wood's equation (Wood, 1932), where the mixture is treated as a homogeneous medium with high density and compressibility. Such a plume, when modeled as a sphere, possesses a fundamental frequency, $f_{r}$,

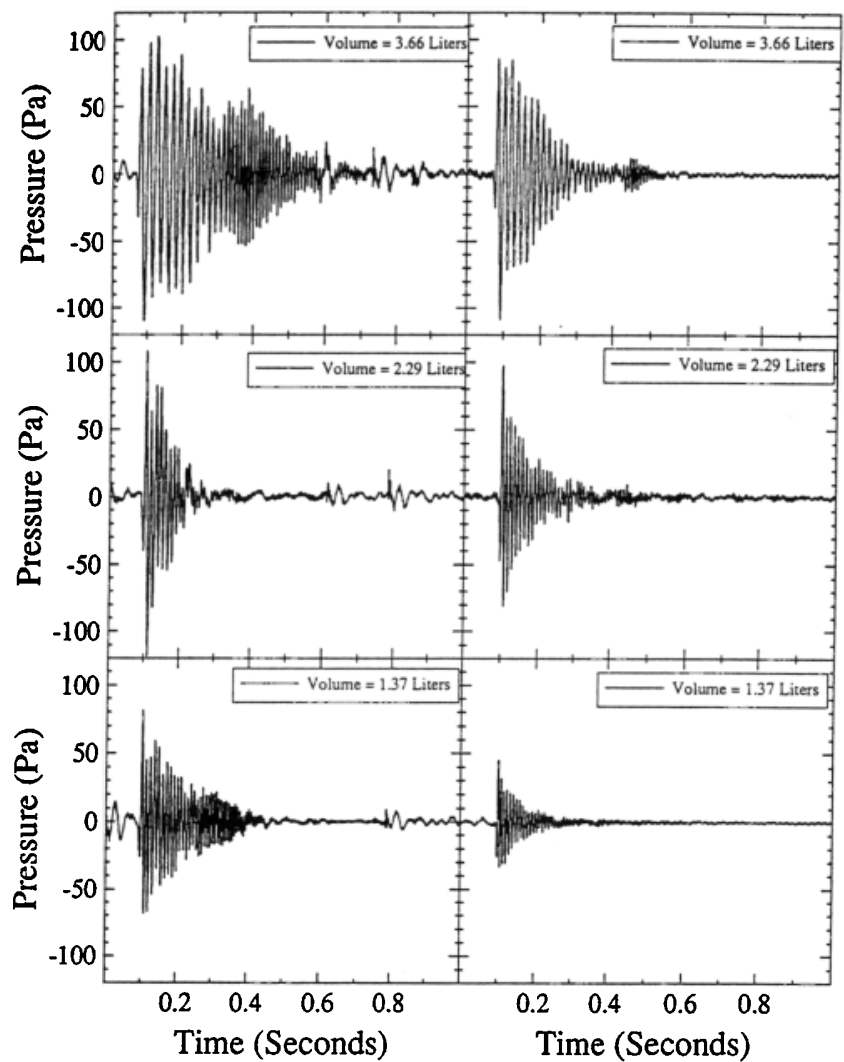

FIG. 3. Pressure-time traces of bubble plume emissions generated by releasing various water volumes onto a still-water surface. The cylinder radius was $5.4 \mathrm{~cm}$ with a suspension height of $15 \mathrm{~cm}$.

given by a modified Minnaert equation (Minnaert, 1933; Carey et al., 1993),

$$
f_{r}=\frac{1}{2 \pi R} \sqrt{\frac{3 P_{0}}{\beta \rho_{0}}},
$$

where $R$ is the plume radius, $P_{0}$ is the ambient pressure, $\rho_{0}$ is the liquid density, and $\beta$ is the void fraction. In the laboratory study, this equation exhibited good agreement with the measured resonance frequencies for void fractions ranging from $32 \%-73 \%$. [We were unable to measure the void fraction at Lake Washington and Puget Sound due to difficulties associated with adapting the measurement procedure (Kolaini et al., 1993) to the field.] It is important to stress the low-frequency acoustical behavior of the plume is governed by its size and void fraction. The individual bubbles and bubble size distribution do not play a prominent role in the collective oscillations of the plume.

Typical pressure-time traces of bubble plume emissions are shown in Fig. 3. These bubble plumes were generated by releasing varying volumes of water from a height of $15 \mathrm{~cm}$. The cylinder was filled with water volumes of $3.66,2.29$, and 1.4 liters, respectively using both fresh and salt water. The sudden onset of low-frequency acoustic emission is well correlated with the detachment of the bubble plume, and the decay in the emission evident in all the traces is reminiscent of the ring-down of a freely oscillating bubble. Note that the decay rate is greater for plumes generated in salt water than in fresh water. 


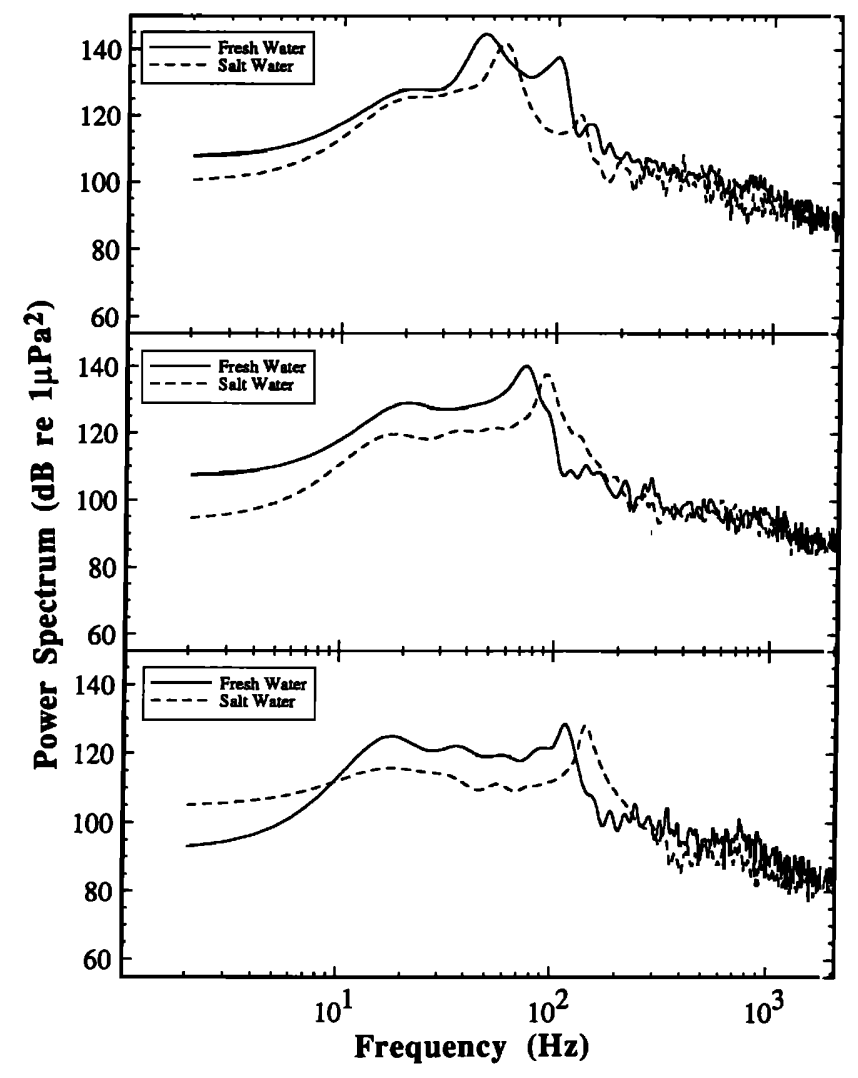

FIG. 4. The power spectral levels of the acoustic emissions shown in Fig. 3.

The corresponding acoustic power spectra are shown in Fig. 4. Prominent low-frequency peaks are evident in all instances, with the "salt-water" peaks occurring at slightly higher frequencies. Moreover, the low-frequency emissions observed in salt water are 3-5 dB lower than for plumes in fresh water. Measured peak frequencies, peak spectral levels and detached plume radii for the events depicted in Figs. 3 and 4 are given in Table I. The data clearly show that the detached plume radius decreases and the fundamental resonance frequency increases with decreasing water volume.

Figure 5 is a plot of the detached plume radius $R$, as a function of the fundamental frequency of the acoustic emission. Experimental measurements of bubble plumes of various sizes and resonance frequencies, both in fresh and salt water, are shown in this figure. These measurements exhibit reasonable agreement with theoretical predictions calculated using Eq. (2) and void fraction ranging from $30 \%-60 \%$. Note that these bounds are consistent with the range of void fraction measured previously in the laboratory.

The sizes of the bubble plumes generated in salt water are typically $10 \%-15 \%$ smaller than those produced in fresh

TABLE I. Characteristics of low-frequency acoustic emissions depicted in Figs. 3 and 4.

\begin{tabular}{lcccccc}
\hline \hline & \multicolumn{3}{c}{ Fresh water } & \multicolumn{3}{c}{ Salt water } \\
\hline Radius & $10.1 \mathrm{~cm}$ & $8.5 \mathrm{~cm}$ & $4.3 \mathrm{~cm}$ & $8.4 \mathrm{~cm}$ & $7.0 \mathrm{~cm}$ & $3.3 \mathrm{~cm}$ \\
PSL & 145 & 140 & 106 & 141 & 137 & 119 \\
$\left(\mathrm{~dB}\right.$ re: $\left.\mu \mathrm{Pa}^{2}\right)$ & & & & & & \\
$\omega_{0}$ & $44 \mathrm{~Hz}$ & $73 \mathrm{~Hz}$ & $115 \mathrm{~Hz}$ & $57 \mathrm{~Hz}$ & $91 \mathrm{~Hz}$ & $135 \mathrm{~Hz}$ \\
\hline \hline
\end{tabular}

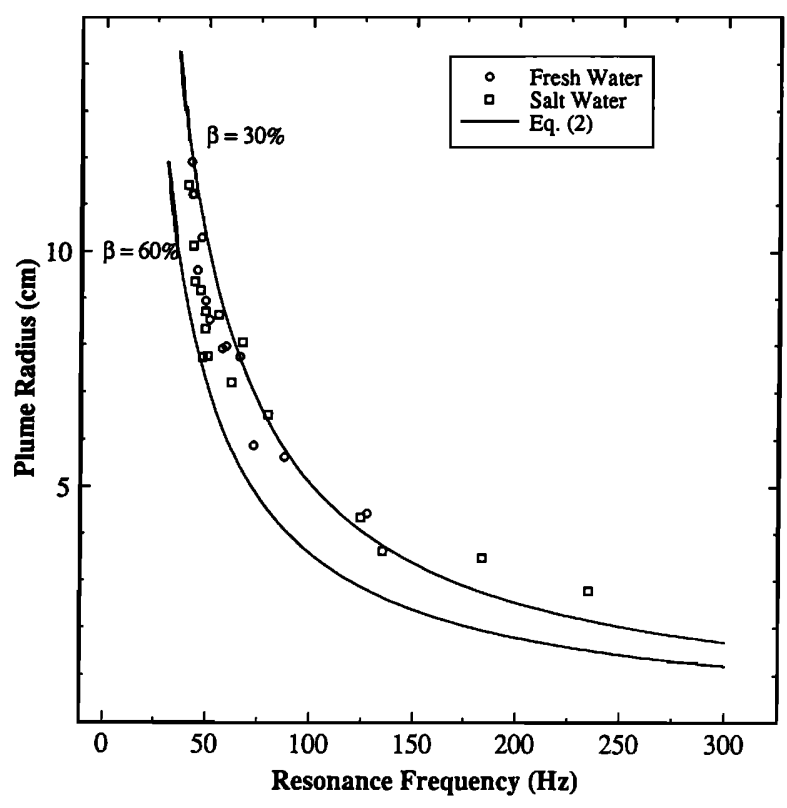

FIG. 5. Bubble plume resonance frequency as a function of plume radius for a variety of different conditions in both fresh and salt water. The solid lines are the theoretical results given by Eq. (2) for void fractions of $30 \%$ and $60 \%$.

water. This decrease in size may have been the reason why the resonance frequencies shifted to higher values. These differences in spectral features evident in fresh and salt water were reproduced in all the measurements. Figure 6 is a plot of the plume resonance frequency versus the volume of the released water obtained from measurements in both fresh and salt water. Larger water volumes produce larger plume sizes with increasing void fraction, therefore, lower resonance frequency. The difference in resonance frequencies of the bubble plumes in salt and fresh water remained approximately constant with increasing water volume.

The data presented in Figs. 3 and 4 correspond to single events that are the characteristic of bubble plume detachment. In their study of the sound production by a tipping

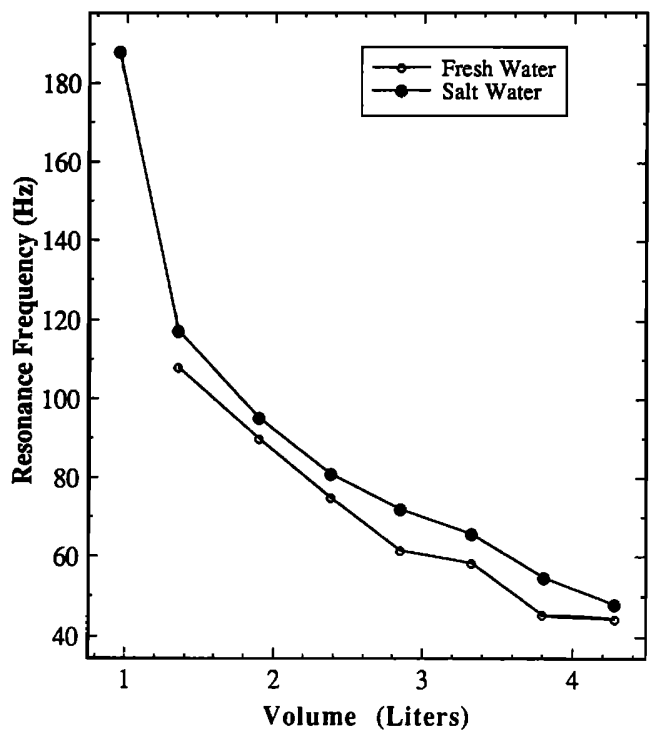

FIG. 6. Bubble plume resonance frequency versus the volume of released water for plumes generated in salt and fresh water. 


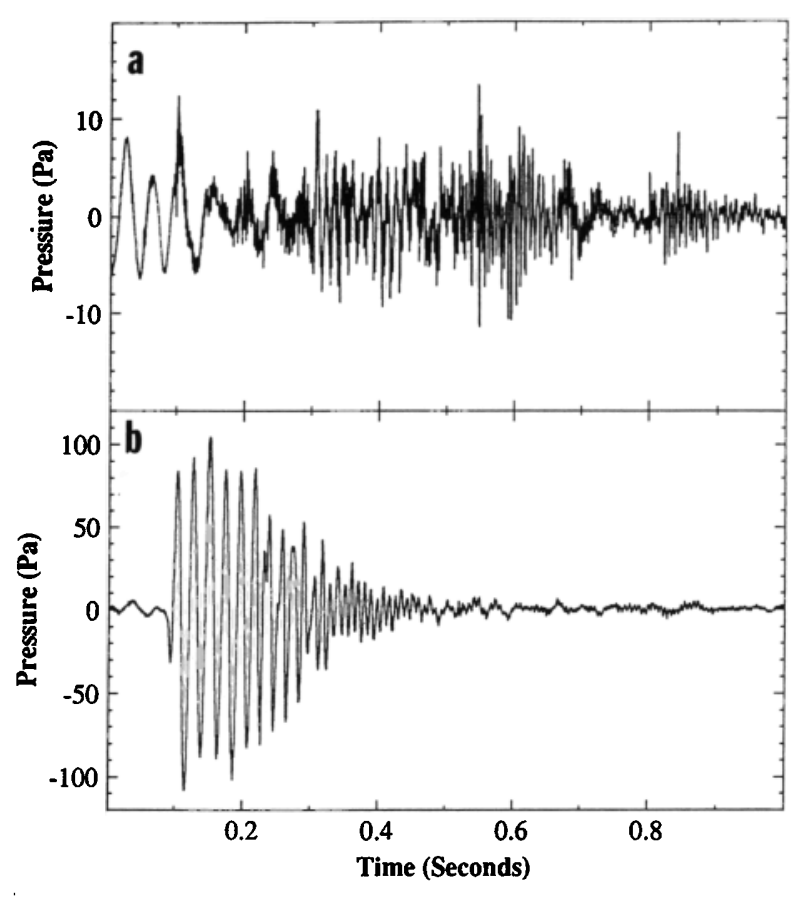

FIG. 7. Representative results of bubble plume acoustic emissions in salt water created by releasing 3.66 liters of water from a cylinder suspension height of $15 \mathrm{~cm}$. (a) The jet was disturbed by a small piece of rubber membrane which partially obstructed the exit opening of the cylinder. The singular low-frequency damped sinusoid is clearly shown together with high-frequency noise. (b) The undisturbed jet.

trough Carey et al. (1993) also reported data showing a pronounced isolated, low-frequency damped sinusoidal characteristic. For our situation, however, the pressure amplitude of the low-frequency signal depends in part on the sink strength located somewhere along the axis of the jet and, contrary to Carey et al. (1993) the bubble size distribution does not play a prominent role. A slight reduction in the level of salt water acoustic emissions was also observed by Carey et al. (1993). This was presumably caused by an increase in the number of small bubbles is salt water which resulted in enhanced thermal damping coupled with an increase in the air/water interface area (Prosperetti, 1988). We believe this to be the case with our experiments as well.

Figure 7(a) and (b) show acoustic emissions produced when $3.66 \mathrm{~L}$ of salt water was released from a height of 15 $\mathrm{cm}$. The physical parameters of the container were the same for Fig. 7(a) and (b) except that, in 7(a), not all of the membrane had retracted from the container opening upon rupture. This changed the jet characteristics from a transient water "slug" to a semi-transient water "pour" impacting the surface. The acoustic "signature" shown in Fig. 7(a), which is typical of irregular bubble-plume separation, is clearly different than the one in Fig. 7(b) for undisturbed flow. Nevertheless, the acoustic event shown in Fig. 7(a), which resembles some of the events that were reported by Carey et al. (1993), indicates the presence of a significant amount of low- as well as high-frequency sound energy. These two examples demonstrate the sensitivity of the acoustic emissions on the complicated nature of the air entrainment processes. In any case, it seems reasonable to suggest that the tipping trough experiments of Carey et al. (1993) also gen- erated low-frequency, high-amplitude sound when a bubbly structure separated from the crater that was created by water impact.

When radiating at its fundamental frequency, the bubble plume possesses a characteristic dimension which is much smaller than the acoustic wavelength; thus it can be regarded as acoustically compact. Such a source is well approximated by a monopole, and the contribution of surface reflection can be accounted for by the method of images. Assuming a planar pressure release surface, lossless low-frequency radiation $(k H \ll 1)$, and far-field propagation $(r \gg H)$, the radiated acoustic pressure is given by

$$
P(r, \theta, t)=P_{\mathrm{ax}} \cos \theta, \quad P_{\mathrm{ax}}=\frac{2 S k H}{r} e^{i(\omega t-k r)},
$$

where $r$ is the distance from a point on the surface immediately above the submerged monopole to the field point, $\theta$ is the angle subtended by $\mathbf{r}$ relative to the dipole axis, $k$ is the wave number, $S$ is the monopole source strength, $H$ is the source depth, and $P_{\mathrm{ax}}$ is the pressure measured vertically downward along the axis of the dipole where $\theta=0^{\circ}$. In such a case the corresponding time-averaged intensity takes on a particularly simple form:

$$
I(r, \theta)=\frac{1}{\rho_{0} c}\left(\frac{1}{\tau} \int_{t_{1}}^{t_{2}} P_{\mathrm{ax}}^{2} d t\right) \cos ^{2} \theta,
$$

where $\tau=t_{1}-t_{2}$ is the duration of the acoustic "event." To obtain the total radiated acoustic energy, we multiply Eq. (4) by $\tau$ and integrate over a hemisphere of radius $r$ centered at the point where the dipole intersects the pressure release surface:

$$
E=\frac{2 \pi r^{2}}{3 \rho_{0} c} \int_{t_{1}}^{t_{2}} P_{\mathrm{ax}}^{2} d t
$$

By measuring the acoustic pressure along the axis of the dipole at a depth $r$, it is possible to determine the total energy radiated in the half-space by the bubble plume pulsating beneath the pressure release surface. In Fig. 8(a) we plot $P_{\mathrm{ax}}$ as a function of distance for a bubble plume of radius $10.2 \mathrm{~cm}$ and resonance frequency of $45 \mathrm{~Hz}$; the $1 / r$ dependence predicted by Eq. (3) is evident. Moreover, in Fig. 8(b) we plot the normalized radiation pattern measured at a radial distance of $1.0 \mathrm{~m}$; note that trials obtained using released water volumes of 4.2 and 3.66 liters both yield the predicted $\cos \theta$ dependence.

The acoustical characteristics of a detached bubble plume depend on the impact velocity of the water jet, which can be easily modified by changing the suspension height of the container. The radiated acoustic pressure produced by a detached plume significantly increases with increasing jet velocity. The initial potential energy of the slug of water is $U=\rho_{0} g V_{w} H_{\mathrm{av}}$, where $V_{w}$ and $H_{\mathrm{av}}$ are the water volume and average height of the container from water-surface, respectively. Figure 9 is a plot of the total radiated acoustic energy, as given in Eq. (5), versus the initial potential energy of the water jet. The speed of sound in the liquid is taken to be constant with a value of $1480 \mathrm{~m} / \mathrm{s}$. The linear fit to data in two different regimes both in salt and fresh water suggests 


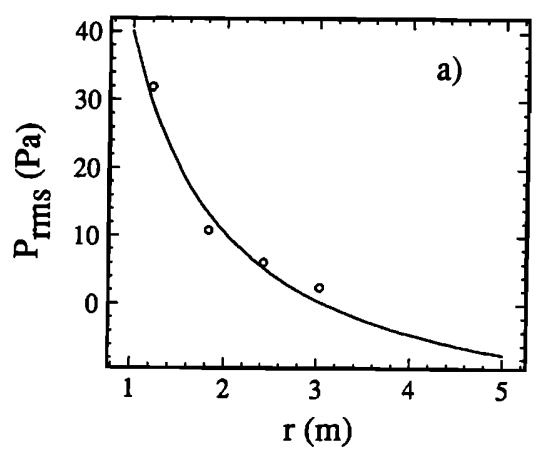

b)

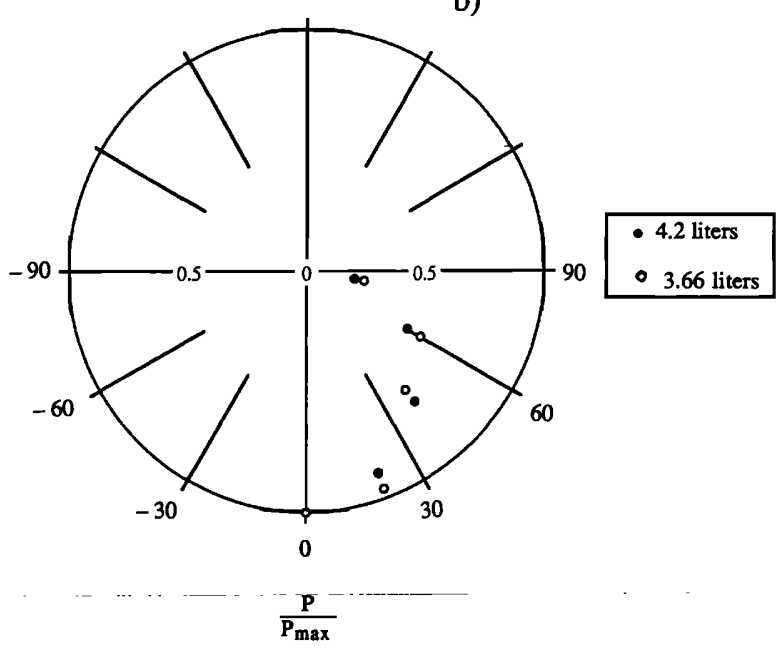

FIG. 8. Radiation characteristics of bubble plumes in salt water. (a) The acoustic pressure as a function of distance for released water volume of 4.2 liters. (b) The normalized rms acoustic pressure as a function of angle for released water volumes of 4.2 and 3.66 liters.

that a fixed fraction of the potential energy of the jet was directly converted to acoustic energy. Consider now the radiation efficiency, which we define as the ratio of the total radiated acoustic energy to the initial potential energy along

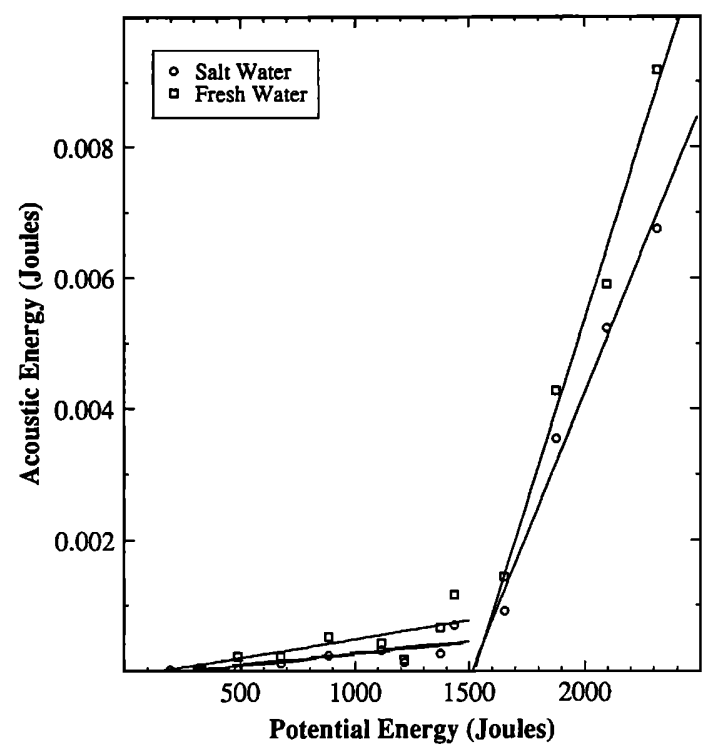

FIG. 9. Variation of the radiated acoustic energy with the initial gravitational potential energy of the impacting water jet in fresh and salt water. the axis of the cylinder. The results show that, for $U<1500$ Joules, $\eta_{\text {eff }}$ is equal to $4.7 \times 10^{-7}$ and $2.6 \times 10^{-7}$ for fresh and salt water, respectively, whereas for $U>1500 \mathrm{~J}$ the corresponding values are $2.6 \times 10^{-6}$ and $2.07 \times 10^{-6}$. The change in slopes of the lines shown in Fig. 9 at a jet potential energy of approximately $1500 \mathrm{~J}$ resemble the transition in the whitecap region to a fully developed sea state. Similar trend has been found in laboratory breaking waves, as measured by Kolaini and Crum (1994). Their result of the radiation efficiency, defined as the ratio of the estimated acoustic energy to the energy of waves prior to breaking, shows values ranging from $0.8 \times 10^{-7}$ to $1.09 \times 10^{-6}$, depending upon the severity of the breaker. Results of laboratory measurements of breaking waves published by Loewen and Melville (1991) show correlation exist between the acoustic radiated energy and mechanical energy dissipated by breaking waves. Kennedy (1992) has shown, from at-sea measurements, that the character of the dipole source strength changes above and below a critical wind friction velocity. These results may have relevance to ocean studies of the acoustic conversion efficiency of breaking gravity waves at high sea states.

\section{CONCLUSIONS}

Some of the features of laboratory experiments designed to measure the acoustic radiation of bubble plumes produced by releasing a various volume of water onto a still-water surface have-been repeated in a free-field environment in both fresh and salt water. These bubble plumes, generally spherical in shape after detachment, undergo volume pulsation very much like a single bubble detaching from an underwater nozzle. The frequency of oscillation of the detached plume depends on the plume volume and void fraction. The measured resonance frequencies of the bubble plumes can be predicted by a modified Minnaert equation for void fractions ranging from $30 \%$ to $60 \%$.

The radiated sound pressure level difference between salt and fresh water plumes was approximately 3-4 dB. Moreover, the power spectra of the acoustic emissions of bubble plumes generated in salt water exhibit a slight shift to higher resonance frequency when compared to spectra obtained in fresh water. This shift was due to the overall reduction in plume sizes produced in salt water. The individual bubbles play an insignificant role in determining the resonance frequency of the plume for frequencies below several tens of $\mathrm{Hz}$. The observed reduction in sound pressure level is most likely due to the increased number of small bubbles is salt water. This effect may be caused by thermal damping coupled with an increase in the total air-water interfacial area.

The physical mechanism that sets the bubble plume into oscillations is the axial jet that is created by sink distributions at the crater region. This mechanism was shown to be identical in both the laboratory measurements reported earlier (Kolaini et al., 1993) and those obtained in the field. The size and resonance frequency of the detached bubble plumes were also similar in the laboratory and field experiments.

We have shown that the radiated acoustic energy is linearly related to the initial potential energy of the cylinder. The conversion efficiencies, defined as the ratio of acoustic 
energy to potential energy, were $4.7 \times 10^{-7}$ and $2.6 \times 10^{-7}$ for fresh and salt water, respectively, and for jet potential energies less than $1500 \mathrm{~J}$. Above $1500 \mathrm{~J}$, the conversion efficiencies increased to $2.6 \times 10^{-6}$ and $2.0 \times 10^{-6}$. The correlation between the acoustic energy and potential energy may have direct relevance to the acoustic remote sensing of sea surface parameters. The change in slopes of the acoustic energy around potential energy of approximately $1500 \mathrm{~J}$ may have some analogy to ocean measurements which indicate a sudden increase in ambient noise levels for wind velocities in excess of some critical value.

\section{ACKNOWLEDGMENTS}

The authors would like to thank NOAA for providing the research launch and access to their facilities located at the Pacific Marine Environmental Laboratory, Seattle, WA. We would like to acknowledge Lt. David Cole for logistic support, Michael Nicholas for assisting in data collection, Alex Ruxton for assisting in data analysis, Lawrence Crum for technical advice, Andrea Prosperetti and William Carey for helpful discussions, and Karl Steffin for his help in launching the experiment. The financial support of the Office of Naval Research and the Office of Naval Technology is greatly appreciated.

Carey, W. M., and Wagstaff, R. A. (1988). "Low-frequency noise fields," J. Acoust. Soc. Am. 80, 1523-1526.

Carey, W. M., Fitzgerald, J. W., Monahan, E. C., and Wang, Q. (1993). "Measurements of the sound produced by a tipping trough with fresh and salt water," J. Acoust. Soc. Am. 93, 3178-3192.

Chapman, N. R., and Cornish, J. W. (1993). "Wind dependence of deep ocean ambient noise at low frequencies," J. Acoust. Soc. Am. 93, 782789.

d'Agostino, L., and Brennen, C. E. (1988). "Acoustical absorption and scattering cross sections of spherical bubble clouds," J. Acoust. Soc. Am. 84, 2126-2134.
Kerman, B. R. (1984). "Underwater Sound Generation by breaking Waves," J. Acoust. Soc. Am. 75, 148.

Kerman, B. R. (1988). Sea Surface Sound: Natural Mechanisms of Surface Generated Noise in the Ocean (Kluwer Academic, Boston).

Kerman, B. R. (1993). Natural Physical Sources of Underwater Sound (Kluwer Academic, Boston).

Knudsen, V. O., Alford, R. S., and Emling, J. W. (1948). "Underwater ambient noise," J. Ocean. Eng. 17, 204-215.

Kennedy, R. M. (1992). "Sea surface dipole sound source dependence on wave-breaking variable," J. Acoust. Soc. Am. 91, 1974-1982.

Kolaini, A. R., and Crum, L. A. (1994). "Observations of underwater sound from laboratory breaking waves and the implications concerning ambient noise in the ocean," J. Acoust. Soc. Am. 96, 1755-1765.

Kolaini, A. R., Roy, R. A., and Crum, L. A. (1991). "An investigation of the acoustic emission from a bubble plume," J. Acoust. Soc. Am. 89, 24522455.

Kolaini, A. R., Roy, R. A. Crum, L. A., and Mao, Y. (1993). "Lowfrequency underwater sound generation by impacting transient cylindrical water jets," J. Acoust. Soc. Am. 94, 2809-2820.

Loewen, M. R., and Melville, W. K. (1991). "Microwave backscatter and acoustic radiation from breaking waves," J. Fluid Mech. 224, 601-623.

Longuet-Higgins, M. S., Kerman, B. R., and Lunde, K. (1991). "The release of an air bubble from an underwater nozzle," J. Fluid Mech. 230, 365390.

Minnaert, M. (1933). "On musical air-bubbles and the sounds of running water," Philos. Mag. 16, 235-248.

Oguz, H. N., and Prosperetti, A. (1991). "Numerical calculation of the underwater noise of rain," J. Fluid Mech. 228, 417-442.

Prosperetti, A. (1988). "Bubble dynamics in oceanic noise," in Sea Surface Sound: Natural Mechanisms of Surface Generated Noise in the Ocean, edited by B. R. Kerman (Kluwer Academic, Boston), pp. 151-171.

Prosperetti, A., Crum, L. A., and Pumphrey, H. C. (1989). "The underwater noise of rain," J. Geophys. Res. 94 (C3), 3255-3259.

Urick, R. J. (1983). Principles of Underwater Sound (McGraw-Hill, New York).

Wenz, G. (1962). "Acoustic ambient noise in the ocean: Spectra and sources," J. Acoust. Soc. Am. 34, 1936-1956.

Wilson, J. H. (1983). "Wind-generated noise modeling," J. Acoust. Soc. Am. 73, 211-216.

Wood, A. B. (1932). A Textbook of Sound (Bell, London). 